\title{
Determinação do melhor método de utilização de um aparelho resistivo medidor de umidade para a madeira de Tectona grandis
}

\author{
Determination of the best method of use of \\ an electric moisture meter for teakwood
}

\author{
Djeison Cesar Batista ${ }^{1}$, Adriano Ribeiro de Mendonça ${ }^{1}$, Adair José Regazzi ${ }^{2}$, \\ Ricardo Jorge Klitzke ${ }^{3}$, Rená Porto Pizetta ${ }^{4}$ e Vinicius Munaldi Lube ${ }^{5}$
}

\begin{abstract}
Resumo
Os medidores elétricos de umidade são amplamente utilizados na indústria madeireira, dada a facilidade de operação, resposta imediata, além de ser um equipamento não destrutivo. O objetivo deste trabalho foi analisar os aspectos operacionais de um aparelho resistivo medidor de umidade, buscando-se determinar o melhor método de utilização para a madeira de teca. Foram delineados 12 tratamentos, derivados dos seguintes fatores analisados: medição da diferença de potencial em relação às fibras (paralela e perpendicular), comprimento dos eletrodos (1/4 e 1/2 da espessura da peça) e escala do aparelho (1, 2 e 3); além do tratamento controle, correspondente ao método gravimétrico. Para a escolha do melhor método de utilização do aparelho aplicou-se uma metodologia específica com a utilização das estatísticas viés $(V)$, média das diferenças absolutas $(M D)$ e erro quadrático médio (EQM); além do teste $t$ de Student para correlação linear ( $r$ e diferenciação de médias (conjuntos de dados pareados) entre a umidade do método gravimétrico e as estimativas de umidade dos demais tratamentos (aparelho elétrico). $O$ melhor método de utilização do medidor elétrico resistivo de umidade consistiu no uso da escala 2, eletrodos de $1 / 4$ da espessura da peça, com a diferença de potencial medida perpendicularmente às fibras (tratamento 3). Este tratamento foi o que permitiu a obtenção de média de umidade mais exata em relação ao método gravimétrico (controle).
\end{abstract}

Palavras-chave: teca, determinação da umidade, relações água-madeira.

\begin{abstract}
Electric moisture meters are widely used in the wood industry because of their easy operation and quick measuring time, besides being non-destructive devices. The aim of this work was to analyze the operational aspects of a resistive moisture meter, in order to achieve the best method of use for teakwood. Twelve treatments were designed, derived from the following analyzed factors: potential difference orientation regarding grain direction (parallel or perpendicular), electrodes length (1/4 and 1/2 of lumber thickness) and meter scale (1, 2 or 3); besides the control treatment (gravimetric method). Some statistics were used to achieve the best method of meter utilization, namely bias, mean absolute difference and mean squared error; and Student's $t$ test for linear correlation $(r)$ and averages differentiation between control and meter treatments. The best method of meter use was scale 2, potential difference measured perpendicular to the grain and fixed at $1 / 4$ of lumber thickness (treatment 3 ). This treatment was the one that obtained the most exact moisture average when compared to the moisture content determined in the gravimetric method (control).
\end{abstract}

Keywords: Tectona grandis, determination of moisture content, wood-water relations.

\footnotetext{
1Professor Adjunto do Departamento de Ciências Florestais e da Madeira. UFES - Universidade Federal do Espírito Santo / Centro de Ciências Agrárias e Engenharias. Avenida Governador Carlos Lindenberg, 316 - Centro - 29550000 - Jerônimo Monteiro, ES, Brasil. E-mail: djeison.batista@ufes.br; adriano.mendonca@ufes.br.

2Professor Visitante do Departamento de Engenharia Florestal e da Madeira. UFES - Universidade Federal do Espírito Santo / Centro de Ciências Agrárias e Engenharias. Avenida Governador Carlos Lindenberg, 316 - Centro - 29550000 - Jerônimo Monteiro, ES, Brasil. E-mail: adairreg@ufv.br.

3Professor Associado do Departamento de Engenharia e Tecnologia Florestal. UFPR - Universidade Federal do Paraná / Centro de Ciências Agrárias. Avenida Professor Lothário Meissner, 632 - Jardim Botânico - 80210-170 - Curitiba, PR, Brasil. E-mail: rjkklitzke@gmail.com.

${ }^{4}$ Engenheira Florestal, Especialista em Engenharia Ambiental. E-mail: renahpizetta@hotmail.com.

${ }^{5}$ Engenheiro Industrial Madeireiro, Mestrando do Departament of Wood Science. UBC - University of British Columbia/ Forest Sciences Centre, 4606 - 2424 Main Mall, V6T 1Z4, British Columbia, Vancouver, Canadá. E-mail: lube@mail.ubc.ca.
}

Sci. For., Piracicaba, v. 44, n. 110, p. 323-329, jun. 2016 DOI: dx.doi.org/10.18671/scifor.v44n110.05 
Batista et al. - Melhor método de utilização de um aparelho

resistivo medidor de umidade para a madeira de Tectona grandis

\section{INTRODUÇÃO}

A teca é uma espécie que vem sendo cultivada no Brasil há algumas décadas e sua madeira possui grande potencial de utilização, principalmente na forma de produtos de maior valor agregado (PMVA), embarcações e lâminas decorativas. De acordo com dados da IBÁ (2014), a área plantada de teca no Brasil em 2013 era de 88.270 ha, o que representou um aumento de 31\% em relação ao ano anterior. Conforme a ABRAF (2013), as florestas de teca em 2012 estavam concentradas principalmente nos estados de Mato Grosso, Pará e Roraima.

Para a geração dos PMVA de madeira sólida mencionados anteriormente, a madeira de teca necessita passar pelo processo de secagem artificial, em que o controle de qualidade da umidade final das cargas é comumente realizado com a utilização de aparelhos elétricos medidores de umidade. Conforme alguns autores (GALVÃO; JANKOWSKY, 1985; SIMPSON, 1991), os medidores de umidade mais populares são os de resistência elétrica, que funcionam pelo princípio de que a resistividade elétrica da madeira varia inversamente com a umidade.

A literatura brasileira é escassa em termos de pesquisas com aparelhos elétricos medidores de umidade, com poucos trabalhos publicados no tema na última década (CALONEGO et al., 2006), ainda mais para a teca, que dentre as essências plantadas é uma espécie menos pesquisada que as de Eucalyptus spp. e Pinus spp.

Sabe-se que a espécie é um fator que influencia significativamente a resistividade da madeira, mas outros fatores também merecem destaque na escolha do método de utilização de um aparelho resistivo medidor de umidade, tais como a posição dos eletrodos em relação às fibras e a profundidade de penetração dos mesmos na madeira (GALINA, 1997; SIMPSON, 1991; SKAAR, 1972; STAMM, 1964).

Dada a relevância da utilização dos aparelhos resistivos na indústria madeireira brasileira para o controle da umidade, e os fatores expostos anteriormente, é fundamental saber o melhor método de utilização de um aparelho para uma determinada espécie, para que as medidas geradas sejam mais exatas.

Foi elaborado o seguinte problema de pesquisa: qual o melhor método de utilização de um aparelho resistivo medidor de umidade para a madeira de teca? A hipótese inicial é que o melhor método será obtido utilizando-se a escala 2 do aparelho, com a diferença de potencial medida entre os eletrodos orientados perpendicularmente à grã e fixados a $1 / 2$ da espessura das peças. Esta hipótese foi elaborada com base nas recomendações de uso do aparelho estabelecidas pelo fabricante (DIGISYSTEM, 2006), exclusive a profundidade de penetração dos eletrodos, conforme explicado na seção material e métodos.

O objetivo deste trabalho foi analisar os aspectos operacionais de um aparelho resistivo medidor de umidade, buscando-se determinar o melhor método de utilização para a madeira de teca.

\section{MATERIAL E MÉTODOS}

Foram utilizadas cinco peças tangenciais de madeira serrada de teca (Tectona grandis L.f.) de dimensões nominais iguais a $40 \times 170 \times 2.200 \mathrm{~mm}$, que foram aleatoriamente amostradas dentre peças empilhadas em um depósito de madeira que fora previamente seca ao ar livre. A umidade média das peças foi igual a 11,5\% correspondendo ao equilíbrio com o ambiente de armazenamento, e encontrava-se dentro da faixa de operação de 8 a 30\% recomendada pelo fabricante do aparelho elétrico resistivo medidor de umidade (DIGISYSTEM, 2006).

\section{Determinação da umidade pelo método do aparelho elétrico}

Foi utilizado um aparelho elétrico medidor de umidade que opera pelo método resistivo, dotado de três escalas de medição. De acordo com as especificações do fabricante, a escala 1 é recomendada para a determinação da umidade de madeiras de densidade maior do que $0,75 \mathrm{~g} . \mathrm{cm}^{-3}$, a escala 2 para densidade entre $0,50 \mathrm{~g} . \mathrm{cm}^{-3} \mathrm{e} 0,75 \mathrm{~g} . \mathrm{cm}^{-3}$, ao passo que a escala 3 para densidade inferior a 0,50 g.cm ${ }^{-3}$ (DIGISYSTEM, 2006). Dessa forma, a escala 2 seria a mais adequada para a determinação da umidade da madeira de teca, porque a mesma apresentou densidade aparente (11,3\% de umidade) média de $0,62 \mathrm{~g}^{\mathrm{cm}} \mathrm{cm}^{-3}$ (método estereométrico). 
Para o modelo do aparelho utilizado, o fabricante disponibiliza apenas dois tipos de eletrodos, com 10 e 20mm de comprimento. Conforme recomendação do fabricante, o comprimento dos eletrodos utilizados deve corresponder a $1 / 3$ da espessura das peças a serem medidas. No entanto, como problema adicional de pesquisa, buscou-se trabalhar com uma peça de $40 \mathrm{~mm}$ de espessura, em que os eletrodos disponíveis não atenderiam ao 1/3 recomendado. Assim, buscou-se observar a viabilidade de utilização do aparelho em uma condição de limitação operacional, que pode ocorrer na prática industrial, supondo-se que o recomendado pelo fabricante reproduza os melhores resultados, não carecendo de validação.

Em cada peça foram demarcados oito corpos de prova de $65 \mathrm{~mm}$ de comprimento, que foram amostrados de forma equidistante ao longo do perfil do comprimento, iniciando-se a amostragem em um dos extremos da peça e encerrando-se no outro extremo. A umidade foi medida na porção mediana dos corpos de prova, primeiro com a diferença de potencial entre os eletrodos, sendo medida na direção perpendicular às fibras, na escala 1 do aparelho, utilizando-se eletrodos de $10 \mathrm{~mm}$ de comprimento ( $1 / 4$ da espessura da peça), trocando-se em seguida para as escalas 2 e 3 . Em seguida, com os mesmos eletrodos, efetuou-se a determinação da umidade com a diferença de potencial na direção paralela à grã, repetindo-se todo o procedimento. De forma idêntica, determinou-se a umidade com os eletrodos de $20 \mathrm{~mm}$ (1/2 da espessura da peça). Ressalta-se que o fabricante recomenda a utilização do aparelho de forma que a diferença de potencial entre os eletrodos seja medida perpendicular às fibras.

\section{Determinação da umidade pelo método gravimétrico}

Em seguida, as peças foram refiladas em serra circular e depois seccionadas transversalmente na posição inicialmente demarcada, produzindo-se 30 corpos de prova de dimensões nominais de $40 \times 60 \times 65 \mathrm{~mm}$ (radial x tangencial x longitudinal). Os corpos de prova foram imediatamente pesados em balança digital semianalítica (exatidão de 0,01g), para obtenção da massa inicial e, em seguida, foram levados à estufa com temperatura de $103 \pm 2{ }^{\circ} \mathrm{C}$, onde permaneceram por 48 horas, até atingirem massa constante (massa anidra), quando foram pesados pela última vez.

Para fins de análise e comparação, os resultados de umidade obtidos pelo método gravimétrico foram considerados como controle, conforme Galvão e Jankowsky (1985). Desta forma, foram comparados os resultados de umidade obtidos com o aparelho elétrico pelos diferentes métodos, com aqueles gerados pelo método gravimétrico, dando-se origem aos tratamentos apresentados na Tabela 1.

Os tratamentos foram elaborados buscando-se compreender o melhor método de utilização do aparelho elétrico medidor de umidade, quanto aos aspectos escala (1, 2 ou 3), medição do diferencial de potencial em relação às fibras (paralela ou perpendicular) e profundidade de penetração dos eletrodos na madeira ( $1 / 4$ ou $1 / 2$ da espessura).

Tabela 1. Tratamentos.

Table 1. Treatments.

\begin{tabular}{|c|c|c|c|c|}
\hline Tratamentos & $\begin{array}{l}\text { Medição da diferença de potencial } \\
\text { em relação à grã }\end{array}$ & Escala & $\begin{array}{l}\text { Profundidade de penetração } \\
\text { dos eletrodos }\end{array}$ & Método \\
\hline 1 (controle) & - & & - & Gravimétrico \\
\hline 2 & & 1 & \multirow{6}{*}{$1 / 4$ da espessura das peças } & \multirow{12}{*}{$\begin{array}{l}\text { Aparelho } \\
\text { elétrico } \\
\text { medidor de } \\
\text { umidade }\end{array}$} \\
\hline 3 & Perpendicular & 2 & & \\
\hline 4 & & 3 & & \\
\hline 5 & & 1 & & \\
\hline 6 & Paralela & 2 & & \\
\hline 7 & & 3 & & \\
\hline 8 & & 1 & \multirow{6}{*}{$1 / 2$ da espessura das peças } & \\
\hline 9 & Perpendicular & 2 & & \\
\hline 10 & & 3 & & \\
\hline 11 & & 1 & & \\
\hline 12 & Paralela & 2 & & \\
\hline 13 & & 3 & & \\
\hline
\end{tabular}


Batista et al. - Melhor método de utilização de um aparelho

resistivo medidor de umidade para a madeira de Tectona grandis

\section{Análise estatística dos dados}

Para análise do melhor método de utilização do aparelho elétrico, foi realizada a comparação das umidades dos tratamentos 2 a 13 com a umidade do método gravimétrico por meio dos seguintes critérios: viés $(V)$, média das diferenças absolutas $(M D)$ e erro quadrático médio (EQM), conforme a Tabela 2.

Tabela 2. Critérios usados para seleção dos melhores tratamentos..

Table 2. Criteria used to select the best treatments.

\begin{tabular}{lc}
\hline Critério & Estimador \\
\hline Viés $(V)$ & $V=\frac{\sum_{i=1}^{n} Y_{i 1}-\sum_{i=1}^{n} Y_{i j}}{n}$ \\
Média das diferenças absolutas $(M D)$ & $M D=\frac{\sum_{i=1}^{n}\left|Y_{i 1}-Y_{i j}\right|}{n}$ \\
Erro quadrático médio $(E Q M)$ & $E Q M=\frac{\sum_{i=1}^{n}\left(Y_{i 1}-Y_{i j}\right)^{2}}{n}$ \\
\hline
\end{tabular}

Em que - $Y_{\mathrm{il}}$ : umidade da madeira (\%) obtida no tratamento I (controle, método gravimétrico), com i $=\mathrm{I}, 2, \ldots, \mathrm{n}=30 ; \mathrm{Y}_{\mathrm{iij}}$ : estimativa da umidade da madeira (\%) obtida pelo tratamento analisado (aparelho elétrico), com j = 2, 3, ..., I 3 .

Com base na análise das estatísticas $V, M D$ e EQM, procedeu-se a ordenação dos tratamentos atribuindo notas de 1 a 12 de acordo com os resultados destas estatísticas obtidas para cada tratamento, segundo o maior ou menor grau de precisão. Foi considerado o tratamento mais preciso aquele que resultou em menor somatório nas notas das três estatísticas (LIMA, 1986; MENDONÇA et al., 2007).

Além dessas estatísticas, estimou-se a correlação linear $(r)$ entre as medidas de umidade do aparelho elétrico (tratamentos 2 a 13) e a medida de umidade do método gravimétrico. Para testar as correlações foi utilizado o teste $t$ de Student. Se o valor absoluto de $t$ calculado for maior ou igual ao valor de $t$ tabelado em nível de significância de 5\%, implica que existe correlação significativa entre os valores medidos com o tratamento em questão (2 a 13) e o método gravimétrico.

Para verificar se existe diferença significativa entre as médias obtidas pelo aparelho elétrico (tratamentos 2 a 13) e o método gravimétrico (tratamento 1), foi realizado o teste $t$ de Student para dados pareados. Se o valor absoluto de $t$ calculado for maior ou igual ao valor de $t$ tabelado ao nível de significância de 5\%, implica que existe diferença significativa entre as medidas de umidade obtidas no tratamento em questão (2 a 13) e o método gravimétrico (tratamento 1).

\section{RESULTADOS E DISCUSSÃO}

Na Tabela 3 encontram-se as estatísticas viés $(V)$, média das diferenças absolutas $(M D)$, erro quadrático médio (EQM), umidade média (U) e coeficiente de variação $(\mathrm{CV})$ para as estimativas de umidade da madeira de teca por tratamento. Na Tabela 4 são apresentadas as notas atribuídas aos tratamentos, com base nas estatísticas $V, M D$ e EQM, para as estimativas de umidade de madeira de teca obtidas pelo aparelho elétrico.

Tabela 3. Estatísticas das estimativas de umidade de madeira de teca obtidas pelo aparelho elétrico medidor de umidade.

Table 3. Statistics estimates of teakwood moisture obtained by the electric moisture meter.

\begin{tabular}{|c|c|c|c|c|c|c|c|c|c|c|c|c|c|}
\hline \multirow{2}{*}{ Estatística } & \multicolumn{13}{|c|}{ Tratamento } \\
\hline & 1 & 2 & 3 & 4 & 5 & 6 & 7 & 8 & 9 & 10 & 11 & 12 & 13 \\
\hline$V$ & - & 2,90 & $-0,44$ & $-4,02$ & 2,76 & $-0,56$ & $-4,21$ & 1,85 & $-1,63$ & $-5,41$ & 1,93 & $-1,49$ & $-5,28$ \\
\hline$M D$ & - & 2,90 & 0,59 & 4,02 & 2,76 & 0,85 & 4,21 & 1,85 & 1,63 & 5,41 & 1,93 & 1,49 & 5,28 \\
\hline$E Q M$ & - & 8,79 & 0,54 & 16,57 & 8,28 & 1,04 & 18,52 & 3,57 & 2,80 & 29,47 & 3,98 & 2,44 & 28,19 \\
\hline U (\%) & 11,37 & 8,47 & 11,81 & 15,39 & 8,61 & 11,93 & 15,58 & 9,52 & 13,00 & 16,78 & 9,44 & 12,86 & 16,65 \\
\hline $\mathrm{CV}(\%)$ & 5,95 & 4,25 & 3,65 & 3,21 & 4,27 & 3,48 & 3,22 & 4,12 & 3,36 & 3,09 & 4,31 & 3,45 & 3,16 \\
\hline
\end{tabular}


Os resultados positivos e negativos da estatística $V$ indicam subestimação e superestimação, respectivamente. Os menores valores das três estatísticas testadas indicam qual tratamento apresenta maior precisão para o objetivo em questão. Por exemplo, o tratamento 3 teve para a estatística $M D$ o valor 0,59 (Tabela 3 ), que quando comparado às $M D$ dos demais tratamentos, a nota atribuída a esse tratamento foi 1 (Tabela 4). Esse valor significa que, considerando a $M D$, o tratamento 3 obteve a melhor estimativa em relação aos demais avaliados, seguido pelos tratamentos 6 (nota 2) e 12 (nota 3), e assim sucessivamente, até chegar ao tratamento de menor precisão (tratamento 10), que obteve a nota 12 .

Tabela 4. Notas atribuídas aos tratamentos com base nas estatísticas viés, média das diferenças absolutas e erro quadrático médio.

Table 4. Treatment grades based on the statistics: bias; mean absolute difference and mean squared error.

\begin{tabular}{|c|c|c|c|c|c|c|c|c|c|c|c|c|}
\hline \multirow{2}{*}{ Estatística } & \multicolumn{12}{|c|}{ Tratamento } \\
\hline & 2 & 3 & 4 & 5 & 6 & 7 & 8 & 9 & 10 & 11 & 12 & 13 \\
\hline$V$ & 8 & 1 & 9 & 7 & 2 & 10 & 5 & 4 & 12 & 6 & 3 & 11 \\
\hline$M D$ & 8 & 1 & 9 & 7 & 2 & 10 & 5 & 4 & 12 & 6 & 3 & 11 \\
\hline$E Q M$ & 8 & 1 & 9 & 7 & 2 & 10 & 5 & 4 & 12 & 6 & 3 & 11 \\
\hline Total & 24 & 3 & 27 & 21 & 6 & 30 & 15 & 12 & 36 & 18 & 9 & 33 \\
\hline
\end{tabular}

Seguindo o procedimento apresentado e analisando-se os dados das Tabelas 3 e 4, considerando-se o somatório das notas atribuídas para as estatísticas $V, M D$ e EQM, notou-se que os resultados seguiram a mesma ordem de precisão em relação aos resultados encontrados para MD (exemplificado anteriormente). Ou seja, o tratamento 3 obteve a melhor estimativa em relação aos demais tratamentos, seguido pelos tratamentos 6 (nota 6), 12 (nota 9) e 9 (nota 12), ao passo que o tratamento 10 obteve a pior estimativa (nota 36). Destaca-se que os quatro tratamentos que utilizaram a escala 2 do aparelho foram os que obtiveram as quatro melhores notas, ao passo que os quatro tratamentos que utilizaram a escala 3 obtiveram a quatro piores notas.

Na Tabela 5 encontram-se os resultados obtidos para o teste $t$ para correlação linear $(r)$ e teste $t$ para dados pareados entre as medidas de umidade do método gravimétrico (tratamento 1) e dos demais tratamentos avaliados (aparelho elétrico).

Tabela 5. Resultados dos testes t para o coeficiente de correlação linear $(r)$ e teste t para dados pareados. Table 5. Results of $t$ tests for coefficient of linear correlation $(r)$ and $t$ test for paired data.

\begin{tabular}{|c|c|c|c|c|c|c|c|c|c|c|c|c|}
\hline \multirow{2}{*}{ Estatística } & \multicolumn{12}{|c|}{ Tratamento } \\
\hline & 2 & 3 & 4 & 5 & 6 & 7 & 8 & 9 & 10 & 11 & 12 & 13 \\
\hline$r$ & $\begin{array}{c}0,44 \\
*\end{array}$ & $\begin{array}{c}0,49 \\
*\end{array}$ & $\begin{array}{c}0,45 \\
*\end{array}$ & $\begin{array}{c}-0,19 \\
\text { ns }\end{array}$ & $\begin{array}{c}-0,22 \\
\text { ns }\end{array}$ & $\begin{array}{c}-0,20 \\
\text { ns }\end{array}$ & $\begin{array}{c}0,83 \\
*\end{array}$ & $\begin{array}{c}0,86 \\
*\end{array}$ & $\begin{array}{c}0,80 \\
*\end{array}$ & $\begin{array}{c}0,67 \\
*\end{array}$ & $\underset{*}{0,72}$ & $\underset{*}{0,63}$ \\
\hline$t_{c}$ & $\begin{array}{c}-26,12 \\
*\end{array}$ & $\begin{array}{c}3,98 \\
*\end{array}$ & $\begin{array}{c}34,93 \\
*\end{array}$ & $\begin{array}{c}-18,25 \\
*\end{array}$ & $\begin{array}{c}3,53 \\
*\end{array}$ & $\begin{array}{c}25,02 \\
*\end{array}$ & $\begin{array}{c}-24,58 \\
*\end{array}$ & $\begin{array}{c}24,04 \\
*\end{array}$ & $\begin{array}{c}72,46 \\
*\end{array}$ & $\begin{array}{c}-21,01 \\
*\end{array}$ & $\begin{array}{c}17,44 \\
*\end{array}$ & $\begin{array}{c}54,23 \\
*\end{array}$ \\
\hline
\end{tabular}

Em que: *, ns: significativo e não significativo a $5 \%$ de probabilidade, respectivamente; $\mathrm{t}_{c}$ : estatística $\mathrm{t}$ calculada.

As melhores correlações, com maiores valores de $r$ e significativos, foram obtidas pelos tratamentos 8 a 13, que utilizaram eletrodos correspondentes a $1 \frac{1}{2}$ da espessura das peças, com destaque para o tratamento 9, com o maior valor de $r(0,86)$. Comparando-se a orientação da medição da diferença de potencial em relação à grã, as melhores correlações foram obtidas para a orientação perpendicular (tratamentos 2 a 4 e 8 a 10). Ressalta-se que os tratamentos 5 a 7, todos com orientação paralela, foram os únicos que apresentaram correlação negativa e não significativa.

Analisando-se o teste $t$ para dados pareados, houve diferença significativa $(\mathrm{p}<0,05)$ entre as médias de umidade obtidas com o aparelho elétrico em todos os tratamentos avaliados em comparação ao método gravimétrico. Isso indicou que todas as médias de umidade obtidas utilizando-se o aparelho elétrico foram diferentes da média obtida pelo método gravimétrico, em que esta foi considerada a umidade real da madeira. Esse resultado foi inesperado e negativo, porque se esperava que dentre os tratamentos delineados, em pelo menos um não houvesse diferença significativa entre as médias dos conjuntos de dados.

Analisando-se os resultados das Tabelas 3, 4 e 5 conjuntamente, percebe-se que o tratamento 3 (diferença de potencial perpendicular à grã e a $1 / 4$ de espessura da peça) foi o mais recomendado 
para a estimativa da umidade da madeira de teca. Apesar de este tratamento ter apresentado baixo valor de $r$ e ter apresentado média diferente estatisticamente $\left(t_{c}=-26,12^{*}\right)$ daquela do gravimétrico, o mesmo apresentou os melhores resultados para as estatísticas $V, M D$ e EQM. Estas estatísticas indicam que a média do tratamento 3 foi a que mais se aproximou da média do método gravimétrico. O fabricante (DIGISYSTEM, 2006) afirma que uma diferença de $\pm 0,5$ ponto percentual é tolerável entre as medidas do aparelho e a do método gravimétrico, para umidade da madeira entre 6 e $12 \%$, e o tratamento 3 foi o único que se enquadrou nesta tolerância (+0,44 pontos percentuais).

Apesar de o tratamento 9 ter apresentado o maior valor de $r$, a média dele também diferiu estatisticamente $\left(t_{c}=24,04^{*}\right)$ do tratamento 1 , e obteve apenas a quarta melhor nota para as estatísticas apresentadas na Tabela 4. Além disso, apresentou diferença de média em relação ao tratamento 1 superior ao tolerável pelo fabricante $(+1,63$ pontos percentuais).

Da mesma forma, embora o tratamento 6 tenha apresentado a segunda melhor nota (Tabela 4), o mesmo apresentou correlação negativa e não significativa $(p>0,05)$ com os resultados obtidos pelo método gravimétrico. Isso indica que não é razoável a indicação deste tratamento para a estimativa da umidade da madeira.

Essa discussão indica que a metodologia aplicada para a determinação do melhor método de utilização do aparelho elétrico $(V, M D, E Q M$, correlação linear e teste $t$ de Student) foi satisfatória, e que as análises foram complementares para a tomada de decisão. Um bom exemplo refere-se aos resultados obtidos no tratamento 6, em que ao utilizar-se somente as notas das estatísticas $V$, MD e $E Q M$, poder-se-ia chegar a uma conclusão equivocada.

Conforme apresentado no item material e métodos, os comprimentos dos eletrodos utilizados para as peças analisadas não estavam de acordo com a recomendação do fabricante, que orienta que os mesmos tenham $1 / 3$ da espessura das peças. Como os eletrodos foram mais curtos e mais longos, respectivamente $1 / 4 e^{1 / 2}$ da espessura das peças, esse pode ter sido um dos motivos porque nenhum dos tratamentos delineados obteve média estatisticamente não diferente do método gravimétrico, conforme o teste $t$ de Student para dados pareados.

\section{CONCLUSÕES}

O melhor método de utilização do medidor elétrico resistivo de umidade foi utilizando-se a escala 2, eletrodos de $1 / 4$ da espessura da peça, com a diferença de potencial medida perpendicularmente às fibras (tratamento 3). Este tratamento foi o que permitiu a obtenção de média de umidade mais exata em relação ao método gravimétrico (controle).

A hipótese inicial do melhor método de utilização do aparelho elétrico para a madeira de teca (Tectona grandis) foi confirmada, ou seja, as recomendações apresentadas pelo fabricante no manual do aparelho foram confiáveis.

A metodologia estatística aplicada mostrou-se útil para a escolha do melhor método de utilização do aparelho elétrico resistivo medidor de umidade.

\section{REFERÊNCIAS BIBLIOGRÁFICAS}

ABRAF. ASSOCIAÇÃO BRASILEIRA DOS PRODUTORES DE FLORESTAS PLANTADAS. Anuário estatístico da ABRAF: ano base 2012. Brasília, 2013. 148 p.

CALONEGO, F. W.; BATISTA, W. R.; SEVERO, E. T. D.; SANTOS, J. E. G.; RIBAS, C. Avaliação do teor de umidade da madeira de Eucalyptus grandis por medidores elétricos resistivos. Revista do Instituto Florestal, São Paulo, v. 18, p. 71-78, dez. 2006.

DIGISYSTEM. Medidor de umidade para madeiras DL 822 \& DUP 8805. 3.ed. Curitiba, 2006. 18 p.

GALINA, I. C. M. Variação da resistência elétrica em madeiras visando o grupamento de espécies. 1997. 109 p. Dissertação (Mestrado em Ciências - Ciência e Tecnologia de Madeiras) - Universidade de São Paulo, Escola Superior de Agricultura "Luiz de Queiroz", Piracicaba, 1997. 
GALVÃO, A. P. M.; JANKOWSKY, I. P. Secagem racional da madeira. São Paulo: Nobel, 1985.111 p.

IBÁ. INDÚSTRIA BRASILEIRA DE ÁRVORES. Relatório Ibá 2014. 2014. Disponível em: < http://www.iba.org/ images/shared/iba 2014 pt.pdf >. Acesso em: 30 jun. 2015.

LIMA, F. S. Análise de funções de "taper" destinadas à avaliação de multiprodutos de árvores de Pinus elliiottii. 1986. 79 p. Dissertação (Mestrado em Engenharia Florestal) - Universidade Federal de Viçosa, Viçosa, 1986.

MENDONÇA, A. R.; SILVA, G. F.; OLIVEIRA, J. T. S.; NOGUEIRA, G. S.; ASSIS, A. L. Avaliação de funções de afilamento visando a otimização de fustes de Eucalyptus sp. para multiprodutos. Cerne, Lavras, v. 13, n. 1, p. 71-82, 2007.

SIMPSON, W.T. Dry kiln operator's manual. Madison: USDA/FS/FPL, 1991. 274 p.

SKAAR, C. Water in Wood. New York: Syracuse University Press, 1972. 218 p.

STAMM, A. J. Wood and cellulose science. New York: The Ronald Press Company, 1964. 549 p.

Recebido em 18/11/2014

Aceito para publicação em 04/11/2015

Sci. For., Piracicaba, v. 44, n. 110, p. 323-329, jun. 2016 DOI: dx.doi.org/10.18671/scifor.v44n110.05 
